VOL. 68 (2003) [57-71]

\title{
PRODUCTS OF THREE IDEMPOTENT TRANSFORMATIONS
}

\author{
R.P. SUlLivan AND RAChel Thomas
}

\begin{abstract}
In 1988 Howie, Robertson and Schein characterised the transformations of a finite set $X$ that can be written as a product of two or of three idempotent transformations of $X$; and in 1989 Saito did the same for products of four idempotents. In 1998 Thomas extended the characterisation of two idempotents to arbitrary sets, and here we characterise products of three idempotents in general. We also define a notion of complexity for transformations of any set and use it to provide a different solution to the three-idempotent problem.
\end{abstract}

\section{INTRODUCTION}

Let $X$ be an arbitrary set and let $T(X)$ denote the semigroup under composition of all (total) transformations of $X$. For finite $X$, the authors of [6] characterised when $\alpha \in T(X)$ can be written as a product of two or of three idempotents in $T(X)$, and later Saito [9] did the same for products of four idempotents in $T(X)$. In [10] Thomas extended some of the earlier work to arbitrary sets and proved a corresponding result for products of two idempotent linear transformations of a vector space. In Section 2, we characterise products of three idempotents in $T(X)$ when $X$ is arbitrary. A more complicated characterisation of such products was provided by Thomas in [11, Chapter 3]. Since it preserves the approach taken in [6] and uses an interesting notion of complexity for transformations defined on an arbitrary set, we outline Thomas' solution to the three-idempotent problem in Section 3.

In [8, Section 3], the authors aimed to determine when a nilpotent with index 2 is a product of two or of three idempotents in certain semigroups of transformations defined on an infinite set. As noted in [10, p. 65], their results are incorrect and in [10, Corollaries 1 and 2], Thomas purported to correct and generalise their result for products of two idempotents. However, her work is partly incorrect. In Section 2, we fill the gap and extend it to cover products of three idempotents in the relevant semigroups.

\section{Received 11th November, 2002}

Research partially supported by the Hungarian National Foundation for Scientific Research (Grant No. 29525). The first author would like to thank Professors L. Marki and R. Wiegandt for their generous assistance during his visit to the Renyi Mathematics Institute, Hungarian Academy of Science, Budapest in August 2002.

Copyright Clearance Centre, Inc. Serial-fee code: 0004-9727/03 \$A2.00+0.00. 


\section{Products of three idempotents in $T(X)$}

In what follows, $Y=A \dot{\cup} B$ means $Y$ is a disjoint union of $A$ and $B$, and we let id $Y$ denote the identity transformation on $Y$. We adopt the convention introduced in $[1$, Volume 2, p. 241]: namely, if $\alpha \in T(X)$, we write

$$
\alpha=\left(\begin{array}{c}
A_{i} \\
x_{i}
\end{array}\right)
$$

and take as understood that the subscript $i$ belongs to some (unmentioned) index set $I$, that the abbreviation $\left\{x_{i}\right\}$ denotes $\left\{x_{i}: i \in I\right\}$, and that $X \alpha=\left\{x_{i}\right\}$ and $x_{i} \alpha^{-1}=A_{i}$, in which case $X=\bigcup\left\{A_{i}: i \in I\right\}$.

We also let $X \backslash a$ denote the complement of the singleton set $\{a\}$ in $X$ (compare $[1$, Volume 1, p. 67]). Thus, there is usually a substantial difference between the set $X \backslash\left\{a_{i}\right\}$ and the set $X \backslash a_{i}$ (indeed, they are the same if and only if $|I|=1$ ). Likewise, we write $X \cup a$ for the union of $X$ and $\{a\}$ (compare [1, Volume 1, p. 47]).

For convenience we state Thomas' characterisation of products of two idempotents in $T(X)$ [10, Theorem 2]. We say an idempotent $\delta \in T(X)$ is proper if $\delta \neq \mathrm{id}_{X}$ (recall that no injective transformation of $X$ can be a product of proper idempotents).

THEOREM 1. If $X$ is an arbitrary set and $\alpha \in T(X)$ then $\alpha$ is a product of two proper idempotents in $T(X)$ if and only if

(1) $\alpha$ is non-injective, and

(2.1) for each $y \in X \alpha$ such that $y \alpha \neq y$, there exists $x \in X \backslash X \alpha$ such that $x \alpha=y$.

For reference later, note that if $\varepsilon$ is a proper idempotent in $T(X)$ then condition (2.1) is vacuously true for $\varepsilon$ (since $y \varepsilon=y$ for each $y \in X \varepsilon$ ) and this accords with the fact that $\varepsilon=\varepsilon^{2}$, a product of two idempotents.

Each $\alpha \in T(X)$ determines a partition of $X$ (denoted in [1, Volume 1, p. 51] by $\left.\pi_{\alpha}\right)$ : namely,

$$
\operatorname{par} \alpha=\left\{y \alpha^{-1}: y \in X \alpha\right\} .
$$

If $\operatorname{par} \alpha=\left\{A_{i}\right\}$, we say $\left\{a_{i}\right\}$ is a cross-section of $\operatorname{par} \alpha$ if $a_{i} \in A_{i}$ for each $i$.

REMARK. Observe that (2.1) can be phrased more simply as: $X \alpha \backslash$ Fix $\alpha \subseteq(X \backslash X \alpha) \alpha$ where Fix $\alpha=\{x \in X: x \alpha=x\}$. However, to accord with later work we note that if $X \alpha=\left\{b_{i}\right\}$ then (2.1) is equivalent to

(2.2) there is a cross-section $\left\{a_{i}\right\}$ of $\operatorname{par} \alpha$ such that $a_{i} \in(X \backslash X \alpha) \cup b_{i}$ for each $i$.

For, suppose (2.1) holds and let $\operatorname{par} \alpha=\left\{A_{i}\right\}$. Put $J=\left\{i: b_{i} \alpha \neq b_{i}\right\}$ and $K=I \backslash J$. Then (2.1) implies, for each $j$, there exists $a_{j} \in X \backslash X \alpha$ such that $a_{j} \alpha=b_{j}$, thus 
$a_{j} \in A_{j}$ and $a_{j} \in(X \backslash X \alpha) \cup b_{j}$. Also, if we let $a_{k}=b_{k} \in A_{k}$ for each $k$, then $a_{k} \in(X \backslash X \alpha) \cup b_{k}$, and we have a cross-section $\left\{a_{j}: j \in J\right\} \cup\left\{a_{k}: k \in K\right\}$ of par $\alpha$ as in (2.2). Conversely, suppose (2.2) holds and assume $y=b_{i} \neq b_{i} \alpha$. Then $a_{i} \neq b_{i}$, so $x=a_{i} \in X \backslash X \alpha$ and $a_{i} \alpha=b_{i}$, as required for (2.1).

The next result is [4, Lemma 3.8]. In effect, it and its dual enable us to assume that if $\alpha=\lambda \beta \mu$ for some idempotents $\lambda, \mu$ in a regular subsemigroup $S$ of $T(X)$ then $\operatorname{par} \lambda=\operatorname{par} \alpha$ and $X \mu=X \alpha$. This is because Green's $\mathcal{R}$ and $\mathcal{L}$ relations on $T(X)$ are well-known [1, Volume 1, Lemmas 2.5 and 2.6]; and, by Hall's Theorem [3, Proposition II.4.5], the $\mathcal{R}$ and $\mathcal{L}$ relations on $S$ are just the restrictions to $S$ of the corresponding ones on $T(X)$.

LemMa 1. Let $S$ be a regular semigroup. If $a \in S$ and $a=e x$ for some $e^{2}$ $=e \in S$ and $x \in S$ then $a=f x$ for some $f^{2}=f \in S$ such that $f \mathcal{R} a$.

THEOREM 2. Suppose $X$ is an arbitrary set and let $\alpha \in T(X)$ have range $\left\{b_{i}\right\}$. Then $\alpha$ is a product of three proper idempotents in $T(X)$ if and only if

(1) $\alpha$ is non-injective, and

(3.1) there is a cross-section $\left\{a_{i}\right\}$ of $\operatorname{par} \alpha$ such that, if $K=\left\{i \in I: a_{i}\right.$ $\left.\in X \alpha \backslash b_{i}\right\}$, then there exist distinct $c_{k} \in X \backslash\left[\left(X \alpha \backslash b_{k}\right) \cup\left\{a_{i}\right\}\right]$ for each $k \in K$.

Proof: Suppose $\alpha=\delta_{1} \delta_{2} \delta_{3}$ for some idempotents $\delta_{1}, \delta_{2}, \delta_{3}$ in $T(X)$. By Lemma 1, we can assume $\operatorname{par} \delta_{1}=\operatorname{par} \alpha=\left\{A_{i}\right\}$ say, and write

$$
\delta_{1}=\left(\begin{array}{c}
A_{i} \\
a_{i}
\end{array}\right), \quad \delta_{2}=\left(\begin{array}{cc}
\left\{a_{i}, c_{i}\right\} & Y \\
c_{i} & y
\end{array}\right), \quad \delta_{3}=\left(\begin{array}{cc}
\left\{b_{i}, c_{i}\right\} & Z \\
b_{i} & z
\end{array}\right) .
$$

In this notation, $X \delta_{1}=\left\{a_{i}\right\}$ and thus $a_{i} \in A_{i}$ for each $i$; possibly $a_{i}=c_{i}$ and/or $b_{j}=c_{j}$ for some $i$ and $j$; and $y \in Y=X \backslash \bigcup_{I}\left\{a_{i}, c_{i}\right\}$ (if this set is non-empty) and similarly $z \in Z=X \backslash \bigcup_{I}\left\{b_{i}, c_{i}\right\}$. In other words, if necessary, we can redefine the initial $\delta_{1}, \delta_{2}, \delta_{3}$ so that $\alpha$ equals the product of the $\delta_{1}, \delta_{2}, \delta_{3}$ specified in (1).

Let $K=\left\{i \in I: a_{i} \in X \alpha \backslash b_{i}\right\}$. Note that for each $i \in I, c_{i} \notin\left\{a_{j}: j \neq i\right\}$ since $c_{j}=a_{j}$ for $j \neq i$ implies $c_{i}=c_{i} \delta_{2}=a_{j} \delta_{2}=c_{j}$, a contradiction. Similarly, $c_{i} \notin\left\{b_{j}: j \neq i\right\}$. Now if $c_{k}=a_{k}$ for some $k \in K$ then

$$
c_{k} \in X \alpha \backslash b_{k}=\left\{b_{i}: i \neq k\right\}
$$

another contradiction. Therefore, $c_{k} \neq a_{k}$ for each $k \in K$ and thus $c_{k} \notin\left\{a_{i}\right\}$ for each $k$. Consequently,

$$
c_{k} \in X \backslash\left[\left(X \alpha \backslash b_{k}\right) \cup\left\{a_{i}\right\}\right]
$$

and clearly all the $c_{k}$ are distinct (since the $b_{k}$ are distinct and $\delta_{3}$ is a mapping). 
Conversely, suppose $\alpha$ satisfies (1) and (3.1). Put $A_{i}=b_{i} \alpha^{-1}$ and $J=I \backslash K$ and define

$$
\delta_{1}=\left(\begin{array}{cc}
A_{j} & A_{k} \\
a_{j} & a_{k}
\end{array}\right), \delta_{2}=\left(\begin{array}{ccc}
a_{j} & \left\{a_{k}, c_{k}\right\} & Y \\
a_{j} & c_{k} & y
\end{array}\right), \delta_{3}=\left(\begin{array}{ccc}
\left\{a_{j}, b_{j}\right\} & \left\{b_{k}, c_{k}\right\} & Z \\
b_{j} & b_{k} & z
\end{array}\right),
$$

where possibly $a_{j}=b_{j}$ and/or $b_{k}=c_{k}$, but in any case

$$
y \in Y=X \backslash\left(\left\{a_{i}\right\} \cup\left\{c_{k}\right\}\right) \text { and } z \in Z=X \backslash\left(\left\{a_{j}, b_{j}\right\} \cup\left\{b_{k}, c_{k}\right\}\right)
$$

Then $\delta_{2}$ is an idempotent which is well-defined since all elements of $\left\{a_{i}\right\} \cup\left\{c_{k}\right\}$ are distinct. Also, $\delta_{3}$ is an idempotent and it is well-defined since $c_{k} \notin\left(X \alpha \backslash b_{k}\right) \cup\left\{a_{i}\right\}$ for each $k \in K$ and $a_{j} \notin\left\{b_{i}: i \neq j\right\}=X \alpha \backslash b_{j}$ for each $j \in J$. Moreover $\alpha=\delta_{1} \delta_{2} \delta_{3}$ as required.

Suppose in (3.1) that $K=\emptyset$ for some cross-section $\left\{a_{i}\right\}$ of $\operatorname{par} \alpha$. This means $a_{i} \notin X \alpha \backslash b_{i}$ for each $i$ and hence $a_{i} \in(X \backslash X \alpha) \cup b_{i}$ for each $i$. That is, $\alpha$ is a product of two proper idempotents by (2.2), and hence also of three proper idempotents. In other words, products of two proper idempotents vacuously satisfy (3.1).

If $\alpha \in T(X)$, we write $r(\alpha)$ for the rank of $\alpha$ (that is, $|X \alpha|)$ and we also write:

$$
\begin{array}{llrl}
D(\alpha) & =X \backslash X \alpha, & d(\alpha) & =|D(\alpha)|, \\
S(\alpha) & =\{x \in X: x \alpha \neq x\}, & s(\alpha)=|S(\alpha)| \\
C(\alpha)=\bigcup\left\{y \alpha^{-1}:\left|y \alpha^{-1}\right| \geq 2\right\}, & c(\alpha)=|C(\alpha)| .
\end{array}
$$

The cardinal numbers $d(\alpha), s(\alpha)$ and $c(\alpha)$ are called, respectively, the defect, shift and collapse of $\alpha$. They were used by Howie [2] to characterise the transformations of $X$ that can be written as a product of idempotents in $T(X)$ when $X$ is infinite (see [4, Lemma 2.10] for a correction to [2, Lemma 7]).

HowIE'S THEOREM. If $X$ is infinite then the semigroup $E(X)$ generated by the proper idempotents in $T(X)$ is the disjoint union of two semigroups:

$$
\begin{aligned}
& V=\left\{\alpha \in T(X): 1 \leq d(\alpha) \leq s(\alpha)<\aleph_{0}\right\} \\
& H=\left\{\alpha \in T(X): d(\alpha)=s(\alpha)=c(\alpha) \geq \aleph_{0}\right\}
\end{aligned}
$$

In [2, pp. 712-714], Howie showed that each element of $H$ is a product of just four idempotents in $H$. Consequently, Theorem 2 and Saito's work [9] indirectly determine products of four idempotent transformations of an arbitrary set. For, if $X$ is infinite and $\alpha \in V$, let $E(\alpha)=S(\alpha) \cup S(\alpha) \alpha$, the so-called essential domain of $\alpha$, and recall that $\alpha$ maps $E(\alpha)$ into itself and fixes each element outside $E(\alpha)$. Hence, in this case, $\alpha \mid E(\alpha)$ is a transformation of a finite set and Saito characterised when such mappings 
are products of four idempotent transformations of the same set (and naturally these idempotents can be easily extended to idempotent transformations of $X$ itself). On the other hand, the $\alpha \in H$ which do not satisfy the conditions of Theorem 2 are precisely those elements of $H$ which can be written as four (and not less than four) idempotents in $H$.

EXAMPLE 1. In [4, pp. 165-166] Howie gave an example of an $\alpha \in T(X)$ which is a product of four (and not less than four) idempotents in $T(X)$. This can be used to illustrate Theorem 2 as follows. Suppose $X=U \dot{U} V \dot{U} W$ where $|U|=|V|=|X|$, choose $v^{\prime} \in V$ and let $\theta: V \rightarrow U$ be a bijection. Define $\alpha \in T(X)$ by

$$
\begin{array}{cl}
u \alpha=v^{\prime}, & \text { for all } u \in U, \\
v \alpha=v \theta, & \text { for all } v \in V, \\
w \alpha=w, & \text { for all } w \in W .
\end{array}
$$

Then $D(\alpha)=V \backslash v^{\prime}, S(\alpha)=U \cup V$ and $C(\alpha)=U$, so $\alpha \in H$ and hence it is a product of four idempotents in $H$. Now $X \alpha=v^{\prime} \cup U \cup W$. Hence if $U=\left\{u_{i}\right\}$ and $v_{i} \alpha=u_{i}$ for each $i$, and if $W=\left\{w_{j}\right\}$, then

$$
\operatorname{par} \alpha=\left\{U,\left\{v_{i}\right\},\left\{w_{j}\right\}: i \in I, j \in J\right\}
$$

where $w_{j} \alpha=w_{j}$ for each $j$. Therefore any cross-section $\left\{a_{i}\right\}$ of par $\alpha$ consists of some $u^{\prime} \in U$ together with each $v_{i}$ and $w_{j}$. Consequently, in the notation of condition (3.1), for each $v_{i} \neq v^{\prime}$ and for each $j$, we have $v_{i} \notin X \alpha \backslash u_{i}$ and $w_{j} \notin X \alpha \backslash w_{j}$. But $u^{\prime} \alpha=v^{\prime}$ and $u^{\prime} \in X \alpha \backslash v^{\prime}$; and also, if $v^{\prime} \alpha=u^{\prime \prime}$ then $v^{\prime} \in X \alpha \backslash u^{\prime \prime}$. In other words, the $K$ defined in (3.1) contains just two elements corresponding to $u^{\prime}, v^{\prime} \in \operatorname{par} \alpha$. Hence if (3.1) holds, there must exist distinct $c_{k}$ for $k=1,2$ such that

$$
c_{k} \in X \backslash\left[\left(X \alpha \backslash b_{k}\right) \cup\left\{a_{i}\right\}\right]
$$

where $b_{1}=v^{\prime}=u^{\prime} \alpha$ and $b_{2}=u^{\prime \prime}=v^{\prime} \alpha$. But clearly for each $u^{\prime} \in U$ we have:

$$
\left(X \alpha \backslash v^{\prime}\right) \cup\left(u^{\prime} \cup V \cup W\right)=X,
$$

hence $c_{1}$ cannot exist. That is, $\alpha$ does not satisfy (3.1), so it is a product of four (and not three) idempotents in $H$.

In [4, Theorem 3.7], Howie showed in particular that if $|X|=m \geq \aleph_{0}$ then the set

$$
Q_{m}=\{\alpha \in T(X): d(\alpha)=s(\alpha)=c(\alpha)=m\}
$$

is a regular idempotent-generated subsemigroup of $T(X)$. Note that $I_{m}=\left\{\alpha \in Q_{m}\right.$ : $r(\alpha)<m\}$ is an ideal of $Q_{m}$. We let $P_{m}$ denote the Rees quotient semigroup $Q_{m} / I_{m}$ 
and identify this with $J_{m} \cup\{0\}$, where $J_{m}=\left\{\alpha \in Q_{m}: r(\alpha)=m\right\}$ and the product of two elements of $J_{m}$ equals 0 if it lies in $I_{m}$. In [7] Marques showed that $P_{m}$ is also a regular idempotent-generated semigroup. In [10, Corollary 1], Thomas showed that condition (2.1) in Theorem 1 characterises products of two idempotents in $Q_{m}$ and in $P_{m}$ (note that these semigroups contain no injective transformations). For the next result, we need [4, Lemma 2.8]: namely, if $\varepsilon \in T(X)$ is idempotent, and the defect, the shift or the collapse of $\varepsilon$ equals $m$, then $\varepsilon \in Q_{m}$.

Corollary 1. Let $S$ equal $Q_{m}$ or $P_{m}$ and suppose $\alpha \in S$ is non-zero. Then $\alpha$ is a product of three idempotents in $S$ if and only if condition (3.1) in Theorem 2 holds for $\alpha$.

Proof: If $\alpha$ is a product of three idempotents in $S$ then this also holds in $T(X)$, hence $\alpha$ satisfies condition (3.1) in Theorem 2 .

Conversely, suppose $\alpha \in Q_{m}$ satisfies condition (3.1). The proof of Theorem 2 shows that $\alpha=\delta_{1} \delta_{2} \delta_{3}$ for some $\delta_{i} \in T(X)$ where $c\left(\delta_{1}\right)=c(\alpha)$ and $d\left(\delta_{3}\right)=d(\alpha)$. Hence $\delta_{1}$ and $\delta_{3}$ belong to $Q_{m}$ by the remark above. Also, using our earlier notation, we see that $D\left(\delta_{1}\right)=\left\{c_{k}\right\} \cup Y$, hence either $|K|=m$ or $|Y|=m$. In both cases, this means $c\left(\delta_{2}\right)=m$ (since $\left\{a_{i}\right\} \cap\left\{c_{k}\right\}=\emptyset$ in the first case) and thus $\delta_{2} \in Q_{m}$ by the same remark as before.

Suppose $\alpha \in P_{m}$ satisfies (3.1) in Theorem 2. Then, from what we have just seen, $\alpha=\delta_{1} \delta_{2} \delta_{3}$ for some $\delta_{i} \in Q_{m}$; and if any $\delta_{i}$ has rank less than $m$, the same is true of $\alpha$ : that is, if $\alpha \in P_{m}$ then each $\delta_{i} \in P_{m}$.

Each element of $Q_{m}$ and of $P_{m}$ is a product of four idempotents, and the number ' 4 ' is best possible (see [4, Theorem 3.7] and [7, Theorem 3.7]). Hence the above result indirectly determines the elements in these semigroups which are products of four but not three idempotents.

As noted in [8, p. 403], for any infinite cardinal $m$, the sets

$$
\begin{aligned}
K_{m} & =\left\{\alpha \in P_{m}:\left|y \alpha^{-1}\right|=m \text { for some } y \in X\right\} \cup\{0\}, \\
L_{m} & =\left\{\alpha \in P_{m}: \text { for all } p<m, \text { there exists } y \in X \text { such that }\left|y \alpha^{-1}\right|>p\right\} \cup\{0\},
\end{aligned}
$$

are 0-bisimple regular subsemigroups of $P_{m}$ such that $K_{m} \subseteq L_{m}$. In [10, Corollary 2], Thomas purports to show that condition (2.1) in Theorem 1 also characterises products of two idempotents in these semigroups. However her proof is invalid: the $\mu^{\prime}$ defined in $[10$, p. 65$]$ is not idempotent in general.

EXAMPLE 2. Let $X$ denote the set of non-negative integers (so $m=\aleph_{0}$ ) and define $\alpha: X \rightarrow X$ by

$$
\alpha=\left(\begin{array}{ccc}
\{4 n, 4 n+1\} & 4 n+2 & \{4 n+3\} \\
4 n+3 & 4 n & 3
\end{array}\right)
$$


where $n \geq 0$. That is,

$$
\alpha=\left(\begin{array}{ccccccccc}
\{0,1\} & \{4,5\} & \{8,9\} & \ldots & 2 & 6 & 10 & \ldots & \{3,7,11, \ldots\} \\
3 & 7 & 11 & \ldots & 0 & 4 & 8 & \ldots & 3
\end{array}\right)
$$

Then $\alpha \in K_{m}$ and it is not idempotent. But it satisfies condition (2.1): if $y \in X \alpha$ and $y \alpha \neq y$ then $y=4 n$ for some $n \geq 0$ or $y=4 n+3$ for some $n \geq 1$. And, since

$$
D(\alpha)=\{4 n+1: n \geq 0\} \cup\{4 n+2: n \geq 0\}
$$

there exists $x \in X \backslash X \alpha$ such that $x \alpha=y$. However, if $\alpha=\lambda \mu$ for some idempotents $\lambda, \mu \in K_{m}$ then we can assume $\operatorname{par} \lambda=\operatorname{par} \alpha$ and hence $(4 n+2) \lambda=4 n+2$. Since $\mu^{2}=\mu$, this implies $(4 n+2) \mu=4 n=(4 n) \mu$. In addition, since $\lambda$ is idempotent, $\{4 n, 4 n+1\} \lambda$ must equal $4 n$ or $4 n+1$. But if it equals $4 n$ then $(4 n) \mu=4 n+3$, contradicting what we already know about $\mu$. Therefore, it equals $4 n+1$ and so $(4 n+1) \mu=4 n+3=(4 n+3) \mu$. That is,

$$
\mu=\left(\begin{array}{cc}
\{4 n+1,4 n+3\} & \{4 n, 4 n+2\} \\
4 n+3 & 4 n
\end{array}\right) \notin K_{m} .
$$

In other words, if $\alpha$ is a product of two idempotents in $K_{m}$ then it satisfies condition (2.1) in Theorem 1 but the converse may be false.

We now remedy the situation and prove the following result. As noted in [8, p. 405], the $\mathcal{R}$ and $\mathcal{L}$ relations on $K_{m}$ and $L_{m}$ are essentially the same as those on $T(X)$.

ThEOREM 3. Let $S$ equal $K_{m}$ or $L_{m}$ and suppose $\alpha \in S$ is non-zero and has range $\left\{b_{i}\right\}$. Then $\alpha$ is a product of two idempotents in $S$ if and only if there is a cross-section $\left\{a_{i}\right\}$ of $\operatorname{par} \alpha$ such that $a_{i} \in(X \backslash X \alpha) \cup b_{i}$ for each $i$ and

$$
\left|(X \backslash X \alpha) \backslash\left\{a_{i}: a_{i} \neq b_{i}\right\}\right|=m
$$

Proof: If $\alpha=\lambda \mu$ for some idempotents $\lambda, \mu \in S$ then $\lambda, \mu$ are idempotents in $T(X)$ and so, by Theorem 1, condition (2.1) and its equivalent (2.2) holds for $\alpha$. In addition, by Lemma 1 , we can assume $\operatorname{par} \lambda=\operatorname{par} \alpha=\left\{A_{i}\right\}$ say. Write $X \lambda=\left\{a_{i}\right\}$, which is a cross-section of $\operatorname{par} \lambda$ since $\lambda$ is idempotent. Let $J=\left\{i: a_{i} \neq b_{i}\right\}$ and put $K=I \backslash J$. Then $a_{j} \mu=b_{j}=b_{j} \mu$ for each $j$ (since $\mu^{2}=\mu$ ) and $a_{k} \mu=b_{k}=a_{k}$ for each $k$. Moreover, using the definition of $J$ and $K$, we obtain

$$
X \backslash\left(\left\{a_{i}\right\} \cup\left\{b_{i}\right\}\right)=X \backslash\left(\left\{b_{k}\right\} \cup\left\{b_{j}\right\} \cup\left\{a_{j}\right\}\right)=X \backslash\left(X \alpha \cup\left\{a_{j}\right\}\right)=(X \backslash X \alpha) \backslash\left\{a_{j}\right\}
$$

If this set has cardinal $r<m$ then $\mu$ cannot belong to $K_{m}$ or to $L_{m}$. For, so far we have found only one or two elements in each $b_{i} \mu^{-1}$; hence if (4) does not hold then 
$y \mu^{-1}$ cannot have cardinal $m$ for any $y \in X$. Likewise for $L_{m}$ : if $r<m$ then $y \mu^{-1}$ cannot have cardinal greater than $r$ for any $y \in X$. Thus the condition holds.

Conversely, suppose the condition holds for $\alpha \in S$. Let $J=\left\{i: a_{i} \neq b_{i}\right\}$ and note that, by supposition, $a_{j} \in X \backslash X \alpha$ for each $j \in J$. Put $K=I \backslash J$ and define

$$
\lambda=\left(\begin{array}{cc}
A_{j} & A_{k} \\
a_{j} & a_{k}
\end{array}\right), \quad \mu=\left(\begin{array}{ccc}
\left\{a_{j}, b_{j}\right\} & a_{k} & Y \\
b_{j} & a_{k} & y
\end{array}\right)
$$

where $y \in Y=X \backslash\left(\left\{a_{i}\right\} \cup\left\{b_{i}\right\}\right)=(X \backslash X \alpha) \backslash\left\{a_{j}\right\}$, a set with cardinal $m$ by supposition. Clearly, $\lambda$ and $\mu$ are well-defined idempotents in $S$ and $\alpha=\lambda \mu$.

Returning to the Example, we now see why it failed to be a product of two idempotents in $K_{m}$. For, the only cross-section $\left\{a_{i}\right\}$ of $\alpha$ which satisfies $a_{i} \in(X \backslash X \alpha) \cup b_{i}$ for each $i$, is $\{4 n+1: n \geq 0\} \cup\{4 n+2: n \geq 0\}$ which equals $X \backslash X \alpha$; but $a_{i} \neq b_{i}$ for each $a_{i}$ in this cross-section, hence (4) does not hold for this $\alpha$.

Next we consider products of three idempotents in $K_{m}$ and in $L_{m}$. By careful choice of notation, we see the answer is very similar to what we have already done.

Theorem 4. Let $S$ equal $K_{m}$ or $L_{m}$ and suppose $\alpha \in S$ is non-zero and has range $\left\{b_{i}\right\}$. Then $\alpha$ is a product of three idempotents in $S$ if and only if there is a cross-section $\left\{a_{i}\right\}$ of par $\alpha$ such that

(3.1) if $K=\left\{i \in I: a_{i} \in X \alpha \backslash b_{i}\right\}$ then, for each $k \in K$, there exists an element $c_{k} \in X \backslash\left[\left(X \alpha \backslash b_{k}\right) \cup\left\{a_{i}\right\}\right]$ such that $\left|\left\{c_{k}\right\}\right|=|K|$, and

(3.2) if $L=\left\{i \in I \backslash K: a_{i} \neq b_{i}\right\}$ then $\left|(X \backslash X \alpha) \backslash\left(\left\{a_{\ell}\right\} \cup\left\{c_{k}\right\}\right)\right|=m$.

Proof: If $\alpha=\delta_{1} \delta_{2} \delta_{3}$ for some idempotents $\delta_{i} \in S$ then this also holds in $T(X)$, hence $\alpha$ satisfies condition (3.1) in Theorem 2. In addition, we can assume par $\delta_{1}$ $=\operatorname{par} \alpha=\left\{A_{j}\right\} \cup\left\{A_{k}\right\}$ where $J$ and $K$ are defined in the proof of Theorem 2. If $X \delta_{1}=\left\{a_{j}\right\} \cup\left\{a_{k}\right\}$ then, since the $\delta_{i}$ are idempotent, we have $a_{j} \delta_{2}=a_{j}$ for each $j$ and $a_{k} \delta_{2}=c_{k} \delta_{2}=c_{k}$ (with $c_{k}$ defined as before). This implies $a_{j} \delta_{3}=b_{j}=b_{j} \delta_{3}$ and $c_{k} \delta_{3}=b_{k}=b_{k} \delta_{3}$. Moreover, if $L$ is defined as in (3.2) then

$$
X \backslash\left(\left\{a_{j}, b_{j}\right\} \cup\left\{b_{k}, c_{k}\right\}\right)=X \backslash\left(X \alpha \cup\left\{a_{\ell}\right\} \cup\left\{c_{k}\right\}\right)=(X \backslash X \alpha) \backslash\left(\left\{a_{\ell}\right\} \cup\left\{c_{k}\right\}\right) .
$$

If this set has cardinal less than $m$ then $\delta_{3}$ cannot belong to $K_{m}$ or to $L_{m}$. Hence (3.2) holds.

Conversely, suppose the conditions hold for $\alpha \in S$. Using (3.1) we can define $\delta_{1}, \delta_{2}, \delta_{3}$ as at (2) in the proof of Theorem 2. Now, $D(\alpha)=Y \cup\left\{c_{k}\right\}$ and this set has cardinal $m$ since $\alpha \in P_{m}$. If $|Y|=m$ then $\delta_{2} \in K_{m}$. If $|K|=m$, write $\left\{c_{k}\right\}=\left\{u_{k}\right\} \dot{\cup}\left\{v_{k}\right\}$ and redefine $\delta_{2}$ as

$$
\delta_{2}^{\prime}=\left(\begin{array}{ccc}
a_{j} & \left\{a_{k}, u_{k}\right\} & Y^{\prime} \\
a_{j} & u_{k} & y^{\prime}
\end{array}\right)
$$


where $Y^{\prime} \supseteq\left\{v_{k}\right\}$ and $y^{\prime} \in Y^{\prime}$. Then $\delta_{2}^{\prime} \in K_{m}$. Finally, assuming (3.2) holds, then $\left|(X \backslash X \alpha) \backslash\left(\left\{a_{\ell}\right\} \cup\left\{u_{k}\right\}\right)\right|=m$ if $\delta_{2}$ has to be redefined. In other words, (3.2) ensures that $Z$ (or $Z^{\prime}=Z \cup\left\{v_{k}\right\}$ ) has cardinal $m$ and hence that $\delta_{3} \in K_{m} \subseteq L_{m}$.

In [5] the authors showed that if $m$ is a regular cardinal then $K_{m}$ constitutes all products of nilpotents in $P_{m}$; and in [8, Section 2], the authors showed that $L_{m}$ fills the same role when $m$ is a singular cardinal. As remarked in [8, p. 404], it would be interesting to know whether $K_{m}$ and $L_{m}$ are generated by their idempotents.

\section{COMPLEXITY OF TRANSFORMATIONS}

The characterisation of products of three idempotents in $T(X)$ which was given in [6] for a finite set $X$ differs greatly from that stated in Theorem 2 for any set. In [6] the authors used a measure of the complexity of $\alpha \in T(X)$ defined as follows. An admissible $\alpha$-triple is a set $\left\{x, x \alpha, x \alpha^{2}\right\}$ where $x \in X \backslash X \alpha$ and $x \alpha^{2} \neq x \alpha^{3}$ (thus, no two of $x, x \alpha, x \alpha^{2}$ are equal). Then the complexity of $\alpha$ is defined as:

$$
\operatorname{com} \alpha=\max \{|T|: T \text { is a set of pairwise disjoint admissible } \alpha \text {-triples }\} .
$$

The next result is [6, Theorem 3]. In what follows, we write

$$
\text { Fix } \alpha=X \backslash S(\alpha)=\{x \in X: x \alpha=x\} \text { and } \operatorname{fix} \alpha=\mid \text { Fix } \alpha \mid \text {. }
$$

Theorem 5. Suppose $|X|=n<\aleph_{0}$ and $\alpha \in T(X)$. Then $\alpha$ is a product of three proper idempotents in $T(X)$ if and only if $\alpha$ is non-injective and

$$
2 r(\alpha) \leq n+\operatorname{fix} \alpha+\operatorname{com} \alpha
$$

In [11] Thomas extended the notion of complexity to an arbitrary set $X$ and then used it to characterise products of three idempotents in $T(X)$ in general. We prefer the characterisation given in Theorem 2 since it can be more readily transferred to a vector space setting (something we shall explore in a subsequent paper). However, for interest, we now sketch Thomas' alternative approach to the problem for $T(X)$.

Suppose $X$ is any set and let $Y \subseteq X$. We say $\left\{x, x \alpha, x \alpha^{2}\right\}$ is a $Y$-admissible $\alpha$-triple if $x \in X \backslash X \alpha, x \alpha^{2} \neq x \alpha^{3}$ and $x \alpha, x \alpha^{2} \in Y$. We now define the complexity of $\alpha$ over $Y$ as

$\left.\operatorname{com} \alpha\right|_{Y}=\max \{|T|: T$ is a set of pairwise disjoint $Y$-admissible $\alpha$-triples $\}$, and if there are no $Y$-admissible $\alpha$-triples, we put $\left.\operatorname{com} \alpha\right|_{Y}=0$.

To show this is well-defined, suppose there is at least one $Y$-admissible $\alpha$-triple $t$. Then the set $T=\{t\}$ is trivially a set of pairwise disjoint $Y$-admissible $\alpha$-triples. Let 
$\mathcal{F}$ denote the family of all sets of pairwise disjoint $Y$-admissible $\alpha$-triples and let $\mathcal{C}$ be a chain in $\mathcal{F}$ under $\subseteq$. We assert that $\mathcal{F}$ contains $\bigcup \mathcal{C}=K$ say. For, if $s, t \in K$ then $s \in A$ and $t \in B$ for some $A, B \in \mathcal{C}$ with $A \subseteq B$ (say). Thus $s, t \in B$, hence $s \cap t=\emptyset$ and it follows that $K \in \mathcal{F}$. By Zorn's Lemma, $\mathcal{F}$ contains a maximal element, $T_{M}$ say: that is, for each $T \in \mathcal{F}$, if $T_{M} \subseteq T$ then $T_{M}=T$.

We assert that if all elements of $\mathcal{F}$ are finite then $\left.\operatorname{com} \alpha\right|_{Y}$ exists. This is certainly true if $X$ is finite. However, suppose there are $T_{n} \in \mathcal{F}$ with $n \geq 1$ whose (finite) cardinals increase indefinitely: that is, if $\left|T_{n}\right|=a_{n}$ then $a_{1}<a_{2}<\cdots<a_{n}$ $<\cdots$. Note that $T_{4}$ contains at least four pairwise disjoint $\alpha$-triples, and by Zorn's Lemma there exists $M \in \mathcal{F}$ which is maximal with respect to containing $T_{4}$. By supposition, there exists $n$ such that $|M|<\left|T_{n}\right|$ and hence there exists $t \in T_{n} \backslash M$. From the maximality of $M$, we deduce that $t$ intersects each element of $M$. But this is impossible since $t$ contains just three elements of $X$, whereas $M$ contains at least four pairwise disjoint $\alpha$-triples. Hence, when all elements of $\mathcal{F}$ are finite, it contains one with maximum cardinal, therefore $\left.\operatorname{com} \alpha\right|_{Y}$ is well-defined in this case.

Suppose there is an infinite $T \in \mathcal{F}$ and choose $M \in \mathcal{F}$ maximal with respect to containing $T$. We assert that if $S$ is any element of $\mathcal{F}$ then $|S| \leq|M|$. This is certainly true if $S$ is finite since $M$ is infinite. Therefore suppose $S$ is infinite and $|S|>|M|$, so

$$
|S \backslash M|=|S|>|M| \text {. }
$$

Now, for each $s_{i} \in S \backslash M=\left\{s_{i}\right\}$ say, there exists $t_{i} \in M$ such that $s_{i} \cap t_{i} \neq \emptyset$ : otherwise, $s_{i}$ is disjoint with every element of $M$, hence $s_{i} \in M$ (by the maximality of $M)$ which contradicts $s_{i} \in S \backslash M$. Choose $u_{i} \in s_{i} \cap t_{i}$ for each $i$ and note that $u_{i}=u_{j}$ if and only if $i=j$ (since $s_{i}, s_{j} \in S$ are disjoint if $i \neq j$ ). Therefore,

$$
\left|\left\{u_{i}\right\}\right|=|I|=|S \backslash M|=|S| \text {. }
$$

However, if $M=\left\{t_{j}\right\}$ then $|J|=|M|$ and, since $\left\{u_{i}\right\} \subseteq \bigcup M$ and each $t_{j}$ is an $\alpha$-triple, we have:

$$
|S| \leq|\bigcup M| \leq|J|+|J|+|J| \text {. }
$$

Since $M$ is infinite, this implies $|S| \leq|M|$, contradicting our supposition. Therefore the assertion holds: that is, if there exists an infinite $T \in \mathcal{F}$ then all elements of $\mathcal{F}$ have cardinal at most $|M|$ where $M \in \mathcal{F}$ is maximal with respect to containing $T$. Consequently, $\left.\operatorname{com} \alpha\right|_{Y}$ exists in this situation, and in fact it equals $|M|$ for any such $M \in \mathcal{F}$.

Example 3. Suppose $X=\{1,2, \ldots, 8\}$ and let

$$
\alpha=\left(\begin{array}{llllllll}
1 & 2 & 3 & 4 & 5 & 6 & 7 & 8 \\
2 & 3 & 5 & 5 & 6 & 8 & 3 & 8
\end{array}\right) .
$$


If $Y=X$, there are only three $Y$-admissible $\alpha$-triples:

$$
\{1,2,3\},\{4,5,6\},\{3,5,7\} \text {. }
$$

So there are just two maximal sets of pairwise disjoint $Y$-admissible $\alpha$-triples:

$$
T_{1}=\{\{1,2,3\},\{4,5,6\}\} \text { and } T_{2}=\{\{3,5,7\}\} .
$$

That is, when all sets of pairwise disjoint $Y$-admissible $\alpha$-triples are finite, maximal ones can differ in size.

Example 4. Suppose $X=\mathbb{Z}$ and let $A, B, C$ and $D$ denote the sets $1+4 X, 2$ $+4 X, 3+4 X$ and $4 X$, respectively. Define $\alpha \in T(X)$ by

$$
\begin{array}{ll}
a \alpha=a+1 & \text { if } a \in A, \\
b \alpha=b+1 & \text { if } b \in B, \\
c \alpha=c+3 & \text { if } c \in C, \\
d \alpha=d-1 & \text { if } d \in D .
\end{array}
$$

Then $X \backslash X \alpha=A \cup D, A \alpha=B, B \alpha=C, C \alpha=B$ and $D \alpha=C$. Now, if $a \in A$ then $a \in X \backslash X \alpha$ and $a+1=a \alpha \in B, a+2=a \alpha^{2} \in C$ and $a \alpha^{2} \neq a \alpha^{3}$. Therefore

$$
T_{1}=\{\{a, a+1, a+2\}: a \in A\}
$$

is a set of $X$-admissible $\alpha$-triples and it is easy to see they are pairwise disjoint. Moreover, as $a$ ranges over $A$, each element of $B$ and $C$ appears in the second and third components of some triple. Thus, $T_{1}$ is maximal since if $\left\{x, x \alpha, x \alpha^{2}\right\}$ is disjoint with all elements of $T_{1}$ then $x$ must lie in $D$ and hence $x \alpha \in C$, a contradiction. Similarly

$$
T_{2}=\{\{d, d-1, d+2\}: d \in D\}
$$

is a maximal set of $X$-admissible $\alpha$-triples. In fact, $T_{1} \cap T_{2}=\emptyset$ and they have the same cardinal, namely $\aleph_{0}$.

In [11, Chapter 3], Thomas tackles the problem of characterising products of three proper idempotents in $T(X)$ by observing that if $\alpha=\delta_{1} \delta_{2} \delta_{3}$ then $\alpha=\beta \mu$ where $\mu^{2}=\mu$ and $\beta$ is a product of two idempotents. She then uses condition (2.1) and her notion of complexity to examine the nature of $\alpha$. This leads her to define two important subsets of $X$ which are related to $\alpha$ and have interesting combinatorial properties. In turn, these enable her to solve the three-idempotent problem.

To indicate the main steps in this approach, we start with two simple results. In what follows, we let $E$ be the set of all proper idempotents in $T(X)$ and put $E^{2}=\{\lambda \mu: \lambda, \mu \in E\}$. 
LEMmA 2. Suppose $X$ is any set and let $\alpha \in T(X)$. If $\alpha=\beta \mu$ where $\beta$ $\epsilon E^{2}, \mu \in E, \operatorname{par} \beta=\operatorname{par} \alpha$ and $X \mu=X \alpha$ then, for all $x \in X, x \beta \neq x \alpha$ if and only if $x \beta \in X \backslash X \alpha$.

Proof: Clearly, $x \beta \in X \backslash X \alpha$ implies $x \beta \neq x \alpha$. Conversely, if $x \beta \in X \alpha$ then $(x \beta) \mu=x \beta$ (since $\mu$ is idempotent with range $X \alpha$ ) and hence $x \alpha=x \beta$.

LEMmA 3. Suppose $X$ is any set and let $\alpha \in T(X)$. If $\alpha=\beta \mu$ where $\beta$ $\in E^{2}, \mu \in E, \operatorname{par} \beta=\operatorname{par} \alpha$ and $X \mu=X \alpha$ then, for all $x \in X$, if $x \beta \in X \backslash C(\alpha)$ then $x \beta=x$.

Proof: Note that $C(\beta)=C(\alpha)$ since $\operatorname{par} \beta=\operatorname{par} \alpha$. Therefore, if $x \beta \notin C(\alpha)$ then $x \beta \notin C(\beta)$ and hence $(x \beta) \beta=x \beta$ by condition (2.2). Consequently, $x \beta=x$ since $x \beta \notin C(\beta)$.

From the above results, we deduce that if $x \in X$ and $x \beta \in X \backslash C(\alpha)$ then either $x \alpha=x \beta$ (in which case $x \alpha=x$ ) or $x \alpha \neq x \beta$, in which case

$$
x=x \beta \in(X \backslash C(\alpha)) \cap(X \backslash X \alpha)
$$

For any set $X$ and any $\alpha \in T(X)$, we write the image of this last set as

$$
\Theta(\alpha)=[(X \backslash C(\alpha)) \cap(X \backslash X \alpha)] \alpha
$$

and often abbreviate this in context to just $\theta$.

LEMMA 4. For any set $X$ and any $\alpha \in T(X)$, we have

$$
d(\alpha)=|C(\alpha) \backslash X \alpha|+|\Theta(\alpha)|
$$

Proof: Since $\alpha$ is injective on $X \backslash C(\alpha)$, the definition of $\Theta(\alpha)$ implies

$$
|\Theta(\alpha)|=|(X \backslash C(\alpha)) \cap(X \backslash X \alpha)|
$$

Now

$$
\begin{aligned}
X \backslash X \alpha & =(C(\alpha) \backslash X \alpha) \dot{u}[(X \backslash C(\alpha)) \backslash X \alpha] \\
& =(C(\alpha) \backslash X \alpha) \dot{\cup}[(X \backslash C(\alpha)) \cap(X \backslash X \alpha)]
\end{aligned}
$$

and taking the cardinal of each side gives the result.

Next, for any set $X$ and any $\alpha \in T(X)$, we define

$$
\Xi(\alpha)=X \alpha \backslash(\text { Fix } \alpha \cup \Theta \cup \Theta \alpha)
$$


and often abbreviate this in context to just $\Xi$. It follows that

$$
X \alpha=\Xi \cup \operatorname{Fix} \alpha \cup \Theta \cup(\Theta \alpha \backslash \text { Fix } \alpha) .
$$

In fact, the sets on the right of (6) are pairwise disjoint. For, clearly $\Xi$ is disjoint with the others, and Fix $\alpha$ is disjoint with $\Theta \alpha \backslash$ Fix $\alpha$. Also, if $x \in$ Fix $\alpha \cap \Theta$ then $y \alpha^{-1}=\{x\}$ for some $x \in X \backslash X \alpha$ (since $y \in \Theta$ ) and this implies $x=y$ (since $y \in \operatorname{Fix} \alpha$ ), so $x=x \alpha$, a contradiction. Therefore, Fix $\alpha$ and $\Theta$ are disjoint. Finally, if $y \in \Theta \cap \Theta \alpha$ then $y \alpha^{-1}=\{x\}$ for some $x \in X \backslash X \alpha$ and also $y=z \alpha$ for some $z \in \Theta \subseteq X \alpha$. Hence $z \in y \alpha^{-1} \subseteq X \backslash X \alpha$, a contradiction. Therefore, $\Theta$ and $\Theta \alpha$ are disjoint. Thus we have proved the following result.

Lemma 5. For any set $X$ and any $\alpha \in T(X)$, we have

$$
r(\alpha)=|\Xi|+\operatorname{fix} \alpha+|\Theta|+\mid \Theta \alpha \backslash \text { Fix } \alpha \mid .
$$

The importance of $\Xi$ can be glimpsed by noting that if it is empty then $\alpha$ can be written as $\beta \mu$ for some $\beta \in E^{2}$ and $\mu \in E$. For, suppose $\Xi=\emptyset$ and let

$$
\alpha=\left(\begin{array}{c}
A_{i} \\
c_{i}
\end{array}\right) .
$$

Then $\left\{c_{i}\right\}=$ Fix $\alpha \dot{U} \Theta \dot{\cup}(\Theta \alpha \backslash$ Fix $\alpha)$. Let $J=\left\{i \in I: c_{i} \in\right.$ Fix $\left.\alpha \cup \Theta\right\}$ and put $K=I \backslash J$. Choose $a_{k} \in A_{k} \cap \Theta$ for each $k \in K$ and define $\beta \in T(X)$ by

$$
\beta=\left(\begin{array}{cc}
A_{j} & A_{k} \\
c_{j} & a_{k}
\end{array}\right) .
$$

Then $\beta$ is well-defined since no $c_{j}$ equals any $a_{k}$ : for, if $c_{j}=a_{k}$ for some $j, k$ then both $c_{j}$ and $a_{k}$ are in $\Theta$, and $\alpha$ is injective on $X \backslash C(\alpha)$, hence $A_{j}=A_{k}$ and so $j=k$, a contradiction. Also, $\beta$ satisfies condition (2.1): if $y \in X \beta$ and $y \beta \neq y$ then $y$ must lie in $\Theta$ and hence it equals $x \alpha$ for some $x \in X \backslash X \alpha$.

Now define an idempotent $\mu \in T(X)$ by

$$
\mu=\left(\begin{array}{ccc}
c_{j} & \left\{c_{k}, a_{k}\right\} & Y \\
c_{j} & c_{k} & y
\end{array}\right)
$$

where $y \in Y=X \backslash\left(\left\{c_{j}\right\} \cup\left\{c_{k}, a_{k}\right\}\right)$ (if this is non-empty). This is also well-defined: for, in addition, if $a_{k}=c_{\ell}$ for some $k, \ell \in K$ then $c_{\ell} \in \Theta$, contradicting the definition of $K$. Moreover, $\alpha=\beta \mu$ as desired.

In effect, the characterisation in [6] of $\alpha \in E^{3}$ when $X$ is finite uses the complexity of $\alpha$ over all of $X$. The next result establishes a connection between that complexity and the complexity of $\alpha$ over $\Xi$. The proof is long and complicated, so we refer the reader to [11, Lemma 3.5$]$ for the details. 
Theorem 6. For any set $X$ and any $\alpha \in T(X)$, if $\Xi=\Xi(\alpha)$ then

$$
\left.\operatorname{com} \alpha\right|_{X}=|\Theta \alpha \backslash F i x \alpha|+\left.\operatorname{com} \alpha\right|_{\Xi}
$$

This result provides an alternative characterisation of $\alpha \in E^{3}$ when $X$ is finite.

Corollary. Suppose $|X|=n<\aleph_{0}$ and $\alpha \in T(X)$. If $\Xi=\Xi(\alpha)$ then $\alpha$ is a product of three proper idempotents in $T(X)$ if and only if $\alpha$ is non-injective and

$$
|\Xi| \leq|C(\alpha) \backslash X \alpha|+\operatorname{com} \alpha \mid \Xi
$$

Proof: By Theorems 5 and $6, \alpha \in E^{3}$ if and only if $\alpha$ is non-injective and

$$
r(\alpha)-\operatorname{fix} \alpha \leq[n-r(\alpha)]+|\Theta \alpha \backslash \operatorname{Fix} \alpha|+\left.\operatorname{com} \alpha\right|_{\Xi}
$$

which, by Lemma 4 , is equivalent to

$$
r(\alpha)-\operatorname{fix} \alpha-|\Theta \alpha \backslash \operatorname{Fix} \alpha| \leq(|C(\alpha) \backslash X \alpha|+|\Theta|)+\operatorname{com} \alpha \mid \Xi
$$

which in turn, by Lemma 5 , is equivalent to (7).

Earlier we saw that if $\Xi(\alpha)=\emptyset$ then $\alpha$ is a product of three proper idempotents. Clearly this is a very special case of (7) being satisfied by $\alpha$.

Surprisingly, (7) is precisely the condition which characterises products of three idempotents of an arbitrary set. The proof is much longer and more complicated than that of Theorem 6, so again we refer the reader to [11, Theorem 3.3] for the details.

Theorem 7. Suppose $X$ is any set and $\alpha \in T(X)$. Let

$$
\Theta=[(X \backslash C(\alpha)) \cap(X \backslash X \alpha)] \alpha \text { and } \Xi=X \alpha \backslash(\operatorname{Fix} \alpha \cup \Theta \cup \Theta \alpha)
$$

Then $\alpha$ is a product of three proper idempotents in $T(X)$ if and only if $\alpha$ is noninjective and $|\Xi| \leq|C(\alpha) \backslash X \alpha|+\operatorname{com} \alpha \mid \Xi$.

Naturally, the above characterisation must be equivalent to that in Theorem 2 . In [11, Theorem 3.5], Thomas makes some progress towards proving this but the equivalence is not yet entirely clear.

When $X$ is infinite, Howie [2] showed that a product $\alpha$ of idempotents in $T(X)$ is determined by conditions on the cardinals $d(\alpha), s(\alpha)$ and $c(\alpha)$. Clearly, condition (3.1) does not involve cardinals, and the above result has a different character to Howie's original work. It would therefore be interesting to know if $\alpha \in E^{3}$ can be characterised strictly in terms of some simpler cardinality conditions. 


\section{REFERENCES}

[1] A.H. Clifford and G.B. Preston, The algebraic theory of semigroups, Mathematical Surveys (Volume 1 and 2) 7 (American Mathematical Society, Providence, RI, 1961 and 1967).

[2] J.M. Howie, 'The subsemigroup generated by the idempotents of a full transformation semigroup', J. London Math. Soc. 41 (1966), 707-716.

[3] J.M. Howie, An introduction to semigroup theory (Academic Press, London, 1976.).

[4] J.M. Howie, 'Some subsemigroups of infinite full transformation semigroups', Proc. Roy. Soc. Edinburgh Sect. A 88 (1981), 159-167.

[5] J.M. Howie and M.P.O. Marques-Smith, 'A nilpotent-generated semigroup associated with a semigroup of full transformations', Proc. Royal Soc. Edinburgh Sect. A 108 (1988), 181-187.

[6] J.M. Howie, E.F. Robertson and B.M. Schein, 'A combinatorial property of finite full transformation semigroups', Proc. Roy. Soc. Edinburgh Sect. A 109 (1988), 319-328.

[7] M.P.O. Marques, 'A congruence-free semigroup associated with an infinite cardinal number', Proc. Royal Soc. Edinburgh Sect. A 93 (1983), 245-257.

[8] M.P.O. Marques-Smith and R.P. Sullivan, 'Nilpotents and congruences on semigroups of transformations with fixed rank', Proc Royal Soc. Edinburgh Sect. A 125 (1995), 399-412.

[9] T. Saito, 'Products of four idempotents in finite full transformation semigroups', Semigroup Forum 39 (1989), 179-193.

[10] R. Thomas, 'Products of two idempotent transformations over arbitrary sets and vector spaces', Bull. Austral. Math. Soc. 57 (1998), 59-71.

[11] R. Thomas, Products of idempotent transformations, MSc thesis (University of Western Australia, Nedlands, W.A. 1999).

Department of Mathematics and Statistics

University of Western Australia

Nedlands WA 6009

Australia 